\title{
The Effectiveness of Hippotherapy to Recover Gross Motor Function in Children with Cerebral Palsy: A Systematic Review and Meta-Analysis
}

\author{
Laura De Guindos-Sanchez ${ }^{1,2}$, David Lucena-Anton ${ }^{3, *}$ () , Jose A. Moral-Munoz ${ }^{3,4,5}$ (1) and \\ Alejandro Salazar ${ }^{4,5,6} \oplus$, Ines Carmona-Barrientos ${ }^{3}$ \\ 1 Department of Physiotherapy, Upacesur Medical and Functional Rehabilitation Center, 41710 Utrera, Spain; \\ lauraguindos@gmail.com \\ 2 Department of Physiotherapy, Macrosad Early Childhood Care, 41620 Marchena, Spain \\ 3 Department of Nursing and Physiotherapy, University of Cadiz, 11009 Cadiz, Spain; \\ ines.carmona@uca.es (I.C.-B.); joseantonio.moral@uca.es (J.A.M.-M.) \\ 4 Institute of Research and Innovation in Biomedical Sciences of the Province of Cadiz (INiBICA), University \\ of Cadiz, 11009 Cadiz, Spain; alejandro.salazar@uca.es \\ 5 The Observatory of Pain, University of Cadiz, 11009 Cadiz, Spain \\ 6 Department of Statistics and Operational Research, University of Cadiz, 11009 Cadiz, Spain \\ * Correspondence: david.lucena@uca.es
}

Received: 27 July 2020; Accepted: 17 August 2020; Published: 19 August 2020

\begin{abstract}
Cerebral palsy $(\mathrm{CP})$ is a permanent disorder of the posture and movement, which can result in impairments of gross motor function, among others. Hippotherapy (HPT) is an emerging intervention to promote motor recovery in patients with neurological disorders, providing a smooth, precise, rhythmic, and repetitive pattern of movement to the patient. The main objective of this systematic review and meta-analysis of randomized controlled clinical trials was to analyze the effectiveness of HPT interventions on gross motor function in subjects with CP. The following databases were searched in May 2019: PubMed, Scopus, Embase, and Web of Science. The methodological quality of the randomized controlled trials was assessed using the Physiotherapy Evidence Database (PEDro) scale. A total of 10 studies were analyzed in this review, involving 452 participants. Favorable effects were obtained on the gross motor function (Gross Motor Function Measure-66, standardized mean difference $(\mathrm{SMD})=0.81,95 \%$ confidence interval $(\mathrm{CI})=0.47-1.15$, Gross Motor Function Measure-88 dimension A SMD $=0.64,95 \% \mathrm{CI}=0.30-0.97$, dimension B SMD $=0.42,95 \% \mathrm{CI}=0.09-0.75$, and dimension $\mathrm{E} S \mathrm{SMD}=0.40,95 \% \mathrm{CI}=0.06-0.73$ ). The results obtained in the present review show the potential benefit of HPT intervention in improving gross motor function in children with CP.
\end{abstract}

Keywords: hippotherapy; cerebral palsy; equine-assisted therapy; physical therapy; gross motor function

\section{Introduction}

Cerebral palsy (CP) is the main source of physical disability in children [1]. The prevalence of $\mathrm{CP}$ is 2.11/1000 live births since 1985 in high-income developed countries. Children with CP usually present several limitations in terms of postural control, balance, walking, and gross motor function, as well as sensory and perceptual disturbances, spasticity, visual impairment, mental retardation, epilepsy, etc. [2]. These disorders are responsible for inefficient and ineffective movements and activities and it often leads to limitations in carrying out activities of daily living [2]. The neurodevelopmental therapies are usually used in the neurological rehabilitation of children with CP. These therapies are 
focused on decreasing excessive tone, giving the patient a sense of normal position and movement, and easing normal movement patterns [3].

Hippotherapy (HPT) is an equine-assisted therapy that applies the specific movement of horses in the rehabilitation of patients with neurological disorders [4], improving the neurological functions and sensory processes $[5,6]$. The research in HPT has increased in recent years as a complementary therapy to traditional treatments [6]. HPT is based on two main action mechanisms: (i) the transmission of the warmth and (ii) the transmission of three-dimensional movements with rhythmic impulses from the horse to the patient's body. The pelvis of the patient is moved in a repetitive, rhythmic, and soft pattern, which is similar to the movement carried out during human gait. This three-dimensional movement stimulates balance reactions, improves postural balance and the trunk straightening [4]. This therapy provides movements in all the movement planes, coming from the alternating elevation of the horse's back that originate anteversion/retroversion, elevation/decrease, and lateral movement with rotation [5]. In addition, HPT provides sensory input and induces greater postural control and motor responses [6]. Several favorable physical effects of HPT were found in muscle coordination, muscle tone, balance, posture, strength, endurance, and flexibility, improving gait and patterns of abnormal movement. In addition, it also showed positive improvements at the social, cognitive, and psychological levels [6]. Furthermore, several recent reviews suggested that HPT could be effective for the neurological rehabilitation of subjects with CP: Novak et al. stated that HPT was a successful allied health therapy to improve muscle symmetry in subjects with CP [7]; Mendizábal Alonso [8] suggested that HPT was effective to improve postural alignment in subjects with CP; Martin-Valero et al. [9] also reported benefits in the performance of the activities of daily living and quality of life; and Zadnikar and Kastrin [10] also obtained favorable results on postural balance in subjects with $\mathrm{CP}$.

Regarding the motor function of children with $\mathrm{CP}$, the main aim of therapeutic interventions is to increase the performance of the gross motor skills that are key components of the functional mobility [11]. To the best of our knowledge, only a systematic review carried out in 2012 by Whalen and Case-Smith [12] suggested that HPT could produce benefits on gross motor function in subjects with $\mathrm{CP}$. Therefore, the current evidence through meta-analysis analyzing the use of HPT to recover gross motor function in patients with CP is limited. Consequently, the aim of this systematic review and meta-analysis is to evaluate the effectiveness of HPT for improving gross motor function in children with $\mathrm{CP}$.

\section{Materials and Methods}

\subsection{Search Strategy}

The present review was carried out following the preferred reporting items for systematic reviews and meta-analyses (PRISMA) [13] recommendations for systematic reviews. The literature search was carried out using the databases: PubMed, Web of Science (WoS), Scopus, and Embase. The search covered up to May 2019, without a limit in the starting date. It was performed by combining the following keywords: "hippotherapy" and "cerebral palsy". No filters were applied in relation to the publication dates or language, but the results were filtered to obtain only studies that corresponded to randomized clinical trials (RCTs).

\subsection{Selection Criteria}

The articles included in this review met the following inclusion criteria based on the PICOS model: (P) population: subjects diagnosed with CP; (I) intervention: HPT; (C) comparison: with conventional physical therapy intervention or placebo; $(\mathrm{O})$ outcomes: gross motor function; and (S) study design: RCTs. The exclusion criteria were: (I) studies that involved healthy participants; (II) more than one intervention compared in the study; and (III) an intervention performed using HPT simulators. 
In addition, we excluded articles in which the intervention was based on therapeutic riding because the instructors may not always be medical professionals using an interdisciplinary team approach [10].

\subsection{Study Selection Process and Data Extraction}

First, a literature search was conducted in the scientific databases by combining keywords. Afterwards, we identified and excluded the duplicated articles. After this first selection, the titles and abstracts of the articles found were reviewed. Next, a second exclusion process was made of those studies that did not fulfill the inclusion criteria. These articles obtained after this last selection were evaluated in depth to fulfill the specific inclusion criteria. Finally, the studies that form part of this review were included. Two reviewers (L.D.-G.S. and D.L.A.) independently selected, reviewed, and extracted data form the studies. An additional reviewer (I.C.B.) participated in the consensus of the decisions. We extracted the following information from each study: author, year of publication, number of participants from both groups, average age, gender, levels of the gross motor function classification system (GMFCS), type of $\mathrm{CP}$, intervention carried out, frequency, duration, outcomes, measuring instruments, and results.

\subsection{Assessment of the Methodological Quality of the Studies}

The PEDro [14] was used to assess the methodological quality of the studies. This scale comprises different items in terms of the following domains: performance, selection, information, detection, and attribution bases. A higher score shows a higher methodological quality. A study with a PEDro score of 6 or higher is considered as evidence level 1 (6-8 is good; 9-10 is excellent), and a study with a score of 5 or less is considered as evidence level 2 ( $4-5$ is acceptable; $<4$ is poor) [15].

\section{Results}

Once the database searches were completed, using the different keywords, a total of 276 documents were obtained, as shown in Figure 1. Finally, 10 studies met the inclusion criteria for review.

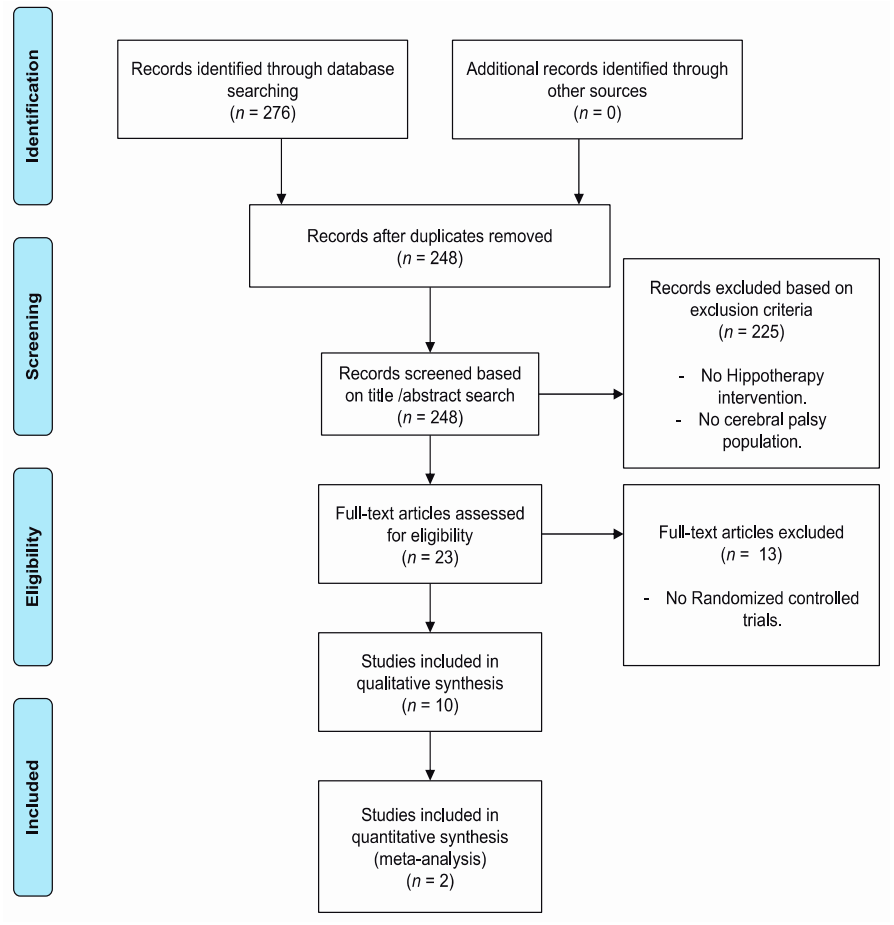

Figure 1. Flow diagram of included articles. 


\subsection{Methodological Quality of the Studies}

Table 1 shows the PEDro scores achieved by the articles reviewed in this study. Three of ten articles were considered to have a high methodological quality: McGibbon et al. [16]; Kwon et al. [17], and Lucena-Antón et al. [5]. Matusiak-Wieczorek et al. [18] achieved the lowest score. The overall methodological quality was acceptable (average total score $=5.1$, range $3-7$ ).

Table 1. Analysis of the methodological quality of the studies (PEDro scores).

\begin{tabular}{ccccccccccccc}
\hline Study & $\mathbf{1}$ & $\mathbf{2}$ & $\mathbf{3}$ & $\mathbf{4}$ & $\mathbf{5}$ & $\mathbf{6}$ & $\mathbf{7}$ & $\mathbf{8}$ & $\mathbf{9}$ & $\mathbf{1 0}$ & $\mathbf{1 1}$ & Total \\
\hline Benda et al., 2003 [19] & - & Yes & Yes & No & No & No & No & Yes & No & Yes & Yes & 5 \\
McGibbon et al., 2009 [16] & - & Yes & Yes & Yes & No & No & Yes & Yes & No & Yes & Yes & 7 \\
Kang et al., 2012 [20] & - & Yes & No & Yes & No & No & No & Yes & No & Yes & Yes & 5 \\
El-Meniawy and Thabet 2012 [21] & - & Yes & No & Yes & No & No & No & No & No & Yes & Yes & 4 \\
Park et al., 2014 [22] & - & Yes & No & Yes & No & No & No & Yes & No & Yes & Yes & 5 \\
Kwon et al., 2015 [17] & - & Yes & No & Yes & Yes & No & Yes & Yes & No & Yes & Yes & 7 \\
Matusiak-Wieczorek et al., 2016 [18] & - & Yes & No & No & No & No & No & No & No & Yes & Yes & 3 \\
Alemdaroglu et al., 2016 [23] & - & Yes & No & Yes & No & No & No & No & No & Yes & Yes & 4 \\
Deutz et al., 2017 [24] & - & Yes & No & Yes & No & No & Yes & No & No & Yes & No & 4 \\
Lucena-Antón et al., 2018 [5] & - & Yes & No & Yes & No & No & Yes & Yes & Yes & Yes & Yes & 7 \\
\hline
\end{tabular}

Range: 0-10. Item 1 is not included in the total score. Item 1: Eligibility criteria; Item 2: Random allocation; Item 3: Concealed allocation; Item 4: Baseline similarity; Item 5: Subject blinding; Item 6: Therapist blinding; Item 7: Assessor blinding; Item 8: >85\% follow up; Item 9: Intention-to-treat analysis; Item 10: Between-group statistical comparison; Item 11: Point and variability measures.

\subsection{Main Characteristics of the Studies Included in the Systematic Review}

Regarding the age of the participants, the highest average age among the control groups was found in the study by McGibbon et al. [16] (8.8 years), while among the intervention groups, it was found in the study by Lucena-Antón et al. [5] (9.6 years). The lowest average age in both groups was presented in the study by Kwon et al. [17] (5.9 and 5.7 years, respectively). In terms of the sample size, the study by Kwon et al. [17] achieved the highest sample size with a total of 91 participants. The overall sample size ranged from 15 to 73 subjects. Table 2 shows the main clinical and demographic characteristics of the participants.

Concerning the different effects analyzed in the different studies, three studies $[17,22,23]$ analyzed the effects of HPT interventions on gross motor function, four studies $[17,18,20,23]$ analyzed the effects on balance, two studies [5,23] analyzed the spasticity, and two studies $[16,19]$ analyzed the muscle activity through electromyography. The main intervention characteristics of the studies included in the systematic review are shown in Table 3. 
Table 2. Main clinical and demographic characteristics of the participants.

\begin{tabular}{|c|c|c|c|c|c|c|}
\hline Study & Participants $(n)$ & Age (Years) \pm SD & Female/Male & GMFCS Levels & $\begin{array}{c}\text { Type: } \\
\text { Diplegia/Hemiplegia } \\
(n)\end{array}$ & Diagnosis \\
\hline Benda et al., 2003 [19] & $\begin{array}{c}\text { IG: }(n=7) \\
\text { CG: }(n=8) \\
\quad N=15\end{array}$ & $4-12$ & ND & ND & ND & Spastic $(n=15)$ \\
\hline \multirow{2}{*}{$\begin{array}{c}\text { McGibbon et al., } 2009 \\
\text { [16] }\end{array}$} & $\begin{array}{c}\text { Phase } 1: \\
\text { IG: }(n=25) \\
C G \cdot(n=19)\end{array}$ & IG: 8.5 & IG: 9/16 & $\begin{array}{l}\text { I: }(n=27) \\
\text { II: }(n=9)\end{array}$ & IG: 12/4 & Spastic $(n=38)$ \\
\hline & $\begin{array}{c}N=44 \\
\text { Phase } 2: \\
\text { IG: }(n=6)\end{array}$ & CG: 8.8 & CG: $11 / 11$ & $\begin{array}{l}\text { III: }(n=5) \\
\text { IV: }(n=6)\end{array}$ & $\begin{array}{c}\text { CG: } 13 / 3 \\
\text { Quadriplegia: } 9\end{array}$ & Mixed $(n=6)$ \\
\hline Kang et al., 2012 [20] & $\begin{array}{c}\text { IG1: }(n=14) \\
\text { IG2: }(n=15) \\
\text { CG: }(n=15) \\
N=44\end{array}$ & $\begin{array}{l}\text { IG1: } 8.2 \pm 1.1 \\
\text { IG2: } 8.2 \pm 1.2 \\
\text { CG: } 7.8 \pm 1.5\end{array}$ & $\begin{array}{l}\text { IG1: } 7 / 7 \\
\text { IG2: } 7 / 8 \\
\text { CG: } 7 / 7\end{array}$ & ND & $\begin{array}{l}\text { IG1: } 5 / 9 \\
\text { IG2: } 5 / 10 \\
\text { CG: } 5 / 9\end{array}$ & ND \\
\hline $\begin{array}{l}\text { El-Meniawy and } \\
\text { Thabet } 2012 \text { [21] }\end{array}$ & $\begin{array}{l}\text { IG: }(n=15) \\
\text { CG: }(n=15) \\
\quad N=30\end{array}$ & $7.02 \pm 0.5$ & ND & ND & ND & Spastic $(n=30)$ \\
\hline \multirow[b]{2}{*}{ Park et al., 2014 [22] } & IG: $(n=34)$ & IG: $6.68 \pm 2.6$ & IG: 19/15 & $\begin{array}{l}\text { I: }(n=14) \\
\text { II: }(n=15)\end{array}$ & IG: $32 / 2$ & \multirow[b]{2}{*}{ Spastic $(n=55)$} \\
\hline & $\begin{array}{c}\text { CG: }(n=21) \\
\qquad N=55\end{array}$ & CG: $7.76 \pm 3.7$ & CG: $11 / 10$ & $\begin{array}{l}\text { III: }(n=11) \\
\text { IV: }(n=15)\end{array}$ & CG: 19/2 & \\
\hline \multirow{2}{*}{ Kwon et al., 2015 [17] } & $\begin{array}{l}\text { IG: }(n=45) \\
\text { CG: }(n=46)\end{array}$ & IG: $5.7 \pm 1.9$ & IG: 25/20 & $\begin{array}{l}\text { I: }(n=24) \\
\text { II: }(n=24)\end{array}$ & IG: $41 / 4$ & \multirow{2}{*}{$\begin{array}{c}\text { Spastic }(n=84) \\
\text { Dyskinetic }(n=4) \\
\text { Ataxic }(n=3)\end{array}$} \\
\hline & $N=91$ & CG: $5.9 \pm 1.8$ & CG: $17 / 29$ & $\begin{array}{l}\text { III: }(n=23) \\
\text { IV: }(n=20)\end{array}$ & CG: $40 / 6$ & \\
\hline \multirow{2}{*}{$\begin{array}{l}\text { Matusiak-Wieczorek } \\
\text { et al., } 2016 \text { [18] }\end{array}$} & IG: $(n=19)$ & IG: $8.42 \pm 2.2$ & IG: $9 / 10$ & $\mathrm{I}:(n=23)$ & IG: $6 / 13$ & \multirow[b]{2}{*}{ Spastic $(n=39)$} \\
\hline & $\begin{array}{c}\text { CG: }(n=20) \\
N=39\end{array}$ & CG: $8.3 \pm 2.6$ & CG: 9/11 & II: $(n=16)$ & CG: 5/15 & \\
\hline
\end{tabular}


Table 2. Cont.

\begin{tabular}{|c|c|c|c|c|c|c|}
\hline Study & Participants $(n)$ & Age (Years) \pm SD & Female/Male & GMFCS Levels & $\begin{array}{c}\text { Type: } \\
\begin{array}{c}\text { Diplegia/Hemiplegia } \\
(n)\end{array}\end{array}$ & Diagnosis \\
\hline $\begin{array}{l}\text { Alemdaroğlu et al., } \\
2016 \text { [23] }\end{array}$ & $\begin{array}{c}\text { IG: }(n=9) \\
\text { CG: }(n=7) \\
N=16\end{array}$ & $7.5 \pm 1.7$ & $7 / 9$ & $\begin{array}{l}\text { IG: I-IV } \\
\text { CG: I-V }\end{array}$ & ND & Spastic $(n=16)$ \\
\hline Deutz et al., 2017 [24] & $\begin{array}{c}\text { IG: }(n=35) \\
\text { CG: }(n=38) \\
N=73\end{array}$ & $\begin{array}{l}\text { IG: } 9.29 \pm 3.7 \\
\text { CG: } 8.87 \pm 2.9\end{array}$ & $\begin{array}{l}\text { IG: } 12 / 23 \\
\text { CG: } 17 / 21\end{array}$ & $\begin{array}{l}\text { II: }(n=27) \\
\text { III: }(n=17) \\
\text { IV: }(n=29)\end{array}$ & $\begin{array}{l}\text { IG: } 35 / 0 \\
\text { CG: } 38 / 0\end{array}$ & Spastic $(n=73)$ \\
\hline $\begin{array}{l}\text { Lucena-Antón et al., } \\
2018 \text { [5] }\end{array}$ & $\begin{array}{c}\text { IG: }(n=22) \\
\text { CG: }(n=22) \\
N=44\end{array}$ & $\begin{array}{l}\text { IG: } 9.5 \pm 2.7 \\
\text { CG: } 8.2 \pm 2.4\end{array}$ & $\begin{array}{l}\text { IG: } 9 / 13 \\
\text { CG: } 7 / 15\end{array}$ & IV-V & ND & Spastic $(n=44)$ \\
\hline
\end{tabular}

CG: control group; GMFCS: Gross Motor Function Classification System; IG: Intervention group; ND: Not described.

Table 3. Summary of interventions carried out by the different studies included in the systematic review.

\begin{tabular}{|c|c|c|c|c|c|c|c|c|}
\hline Study & Participants & Intervention & Frequency & $\begin{array}{c}\text { Session } \\
\text { Duration }\end{array}$ & Total Duration & Outcomes & $\begin{array}{c}\text { Measuring } \\
\text { Instruments }\end{array}$ & Results \\
\hline $\begin{array}{c}\text { Benda et al., } 2003 \\
\text { [19] }\end{array}$ & $\begin{array}{l}\text { IG: }(n=7) \\
\text { CG: }(n=8)\end{array}$ & $\begin{array}{l}\text { IG: HPT } \\
\text { CG: Exercises } \\
\text { on a barrel }\end{array}$ & One session & $8 \mathrm{~min}$ & One session & $\begin{array}{l}\text { Muscle activity in the } \\
\text { paravertebral, hip } \\
\text { abductors/adductors when } \\
\text { sitting, standing, and walking }\end{array}$ & EMG & $\begin{array}{l}\text { IG got better results than CG. Mean } \\
\text { change improvements: } \mathrm{IG}=64.6 \% \\
(\mathrm{SD}=28.3) \text { vs. CG }=-12.8 \%(\mathrm{SD}=88.8) \text {; } \\
\qquad(p=0.051)\end{array}$ \\
\hline \multirow{2}{*}{$\begin{array}{l}\text { McGibbon et al., } \\
2009 \text { [16] }\end{array}$} & $\begin{array}{l}\text { Phase 1: } \\
\text { IG: }(n=25) \\
\text { CG: }(n=19)\end{array}$ & IG: HPT & $\begin{array}{l}\text { Phase 1: } \\
\text { One session }\end{array}$ & Phase 1: $10 \mathrm{~min}$ & $\begin{array}{l}\text { Phase 1: } \\
\text { One session }\end{array}$ & \multirow[t]{2}{*}{ Hip adductors muscle activity } & \multirow{2}{*}{ SEMG } & $\begin{array}{l}\text { Phase 1: The IG significantly improved } \\
\text { the muscle asymmetry of hip adductors } \\
\qquad(p<001 ; d=1.32)\end{array}$ \\
\hline & Phase 2: & $\begin{array}{l}\text { CG: Exercises } \\
\text { on a barrel }\end{array}$ & $\begin{array}{c}\text { Phase 2: } \\
\text { Once a week }\end{array}$ & Phase 2: $40 \mathrm{~min}$ & $\begin{array}{l}\text { Phase 2: } \\
36 \text { weeks }\end{array}$ & & & $\begin{array}{c}\text { Phase 2: After } 12 \text { weeks, } 4 \text { of } 6 \text { children } \\
\text { improved the muscle symmetry of hip } \\
\text { adductors }\end{array}$ \\
\hline
\end{tabular}


Table 3. Cont.

\begin{tabular}{|c|c|c|c|c|c|c|c|c|}
\hline Study & Participants & Intervention & Frequency & $\begin{array}{l}\text { Session } \\
\text { Duration }\end{array}$ & Total Duration & Outcomes & $\begin{array}{l}\text { Measuring } \\
\text { Instruments }\end{array}$ & Results \\
\hline \multirow{2}{*}{$\begin{array}{c}\text { Kang et al., } 2012 \\
\text { [20] }\end{array}$} & $\begin{array}{l}\text { IG1: }(n=15) \\
\text { IG2: }(n=15)\end{array}$ & $\begin{array}{l}\text { IG1: HPT } \\
\text { IG2: PT }\end{array}$ & \multirow{2}{*}{ Once a week } & \multirow{2}{*}{$30 \mathrm{~min}$} & \multirow{2}{*}{8 weeks } & \multirow{2}{*}{ Sitting balance } & \multirow{2}{*}{ Force plate } & \multirow{2}{*}{$\begin{array}{l}\text { The results showed that pathway and } \\
\text { velocity significantly decreased in the HPT } \\
\text { group }(p<0.05) \text { compared to the PT and } \\
\text { CON groups }\end{array}$} \\
\hline & CG: $(n=15)$ & $\begin{array}{l}\text { CG: Non } \\
\text { treatment }\end{array}$ & & & & & & \\
\hline \multirow{2}{*}{$\begin{array}{l}\text { El-Meniawy and } \\
\text { Thabet } 2012[21]\end{array}$} & IG: $(\mathrm{n}=15)$ & IG: HPT & IG: Once a week & IG: $30 \mathrm{~min}$ & \multirow{2}{*}{12 weeks } & \multirow{2}{*}{$\begin{array}{l}\text { Back geometry parameters: } \\
\text { lateral deviation, trunk } \\
\text { imbalance, pelvic tilt, rotation }\end{array}$} & \multirow{2}{*}{$\begin{array}{c}\text { Formetric } \\
\text { instrument } \\
\text { system }\end{array}$} & \multirow{2}{*}{$\begin{array}{l}\text { The results showed improvements in favor } \\
\text { of the IG in all the outcomes }(p<0.05)\end{array}$} \\
\hline & CG: $(n=15)$ & CG: Exercise & $\begin{array}{c}\text { CG: } 3 \\
\text { times/week }\end{array}$ & CG: $1 \mathrm{~h}$ & & & & \\
\hline \multirow{2}{*}{$\begin{array}{c}\text { Park et al., } 2014 \\
\text { [22] }\end{array}$} & & IG: HPT & \multirow[t]{2}{*}{2 times/week } & \multirow[t]{2}{*}{$45 \mathrm{~min}$} & \multirow[t]{2}{*}{8 weeks } & Gross motor function & GMFM-66 & \multirow{2}{*}{$\begin{array}{l}\text { Significant results were obtained in IG } \\
\text { after the intervention compared to the CG: } \\
\text { GMFM- } 66 \text { (all dimensions); GMFM- } 88 \text { (B } \\
\text { and C dimensions); and } 3 \text { domains of the } \\
\text { PEDI-FSS: ( } p<0.05 \text { ) }\end{array}$} \\
\hline & CG: $(n=21)$ & $\begin{array}{l}\text { CG: Non } \\
\text { treatment }\end{array}$ & & & & Functional performance & $\begin{array}{l}\text { GMFM-88 } \\
\text { PEDI-FSS }\end{array}$ & \\
\hline \multirow[t]{2}{*}{$\begin{array}{c}\text { Kwon et al., } 2015 \\
\text { [17] }\end{array}$} & & IG: HPT & \multirow[t]{2}{*}{2 times/week } & \multirow[t]{2}{*}{$30 \mathrm{~min}$} & \multirow[t]{2}{*}{8 weeks } & Gross motor function. & GMFM-66 & \multirow{2}{*}{$\begin{array}{c}\text { Significant results were found between } \\
\text { groups ( } p<0.05) \text { : GMFM- } 66, \text { GMFM- } 88 \\
\text { (total score and dimensions B, C, D, and E). } \\
\text { Moreover, significant results were found } \\
\text { in balance }(p<0.05)\end{array}$} \\
\hline & CG: $(n=46)$ & $\begin{array}{l}\text { CG: Aerobic } \\
\text { exercise }\end{array}$ & & & & Balance & $\begin{array}{l}\text { GMFM- } 88 \\
\text { PBS }\end{array}$ & \\
\hline $\begin{array}{l}\text { Matusiak-Wieczorek } \\
\text { et al., } 2016 \text { [18] }\end{array}$ & $\begin{array}{l}\text { IG: }(n=19) \\
\text { CG: }(n=20)\end{array}$ & $\begin{array}{l}\text { IG: HPT } \\
\text { CG: NI }\end{array}$ & Once a week & $30 \mathrm{~min}$ & 12 weeks & Body balance in sitting position & SAS & $\begin{array}{l}\text { Significant results were obtained in IG for } \\
\text { arm function and control of trunk position: } \\
\qquad(p=0.018)\end{array}$ \\
\hline $\begin{array}{l}\text { Alemdaroğlu et al., } \\
2016[23]\end{array}$ & $\begin{array}{l}\text { IG: }(n=9) \\
\text { CG: }(n=7)\end{array}$ & $\begin{array}{l}\text { IG: HPT } \\
\text { CG: PT }\end{array}$ & $\begin{array}{c}\text { IG: } 2 \text { times/week } \\
\text { CG: } 5 \\
\text { times/week }\end{array}$ & $30 \mathrm{~min}$ & 5 weeks & $\begin{array}{l}\text { Gross motor function, hip } \\
\text { adductors spasticity, balance, } \\
\text { hip abduction angle, knee } \\
\text { distance }\end{array}$ & $\begin{array}{l}\text { GMFMCS } \\
\text { MAS } \\
\text { MFRT } \\
\text { Goniometer }\end{array}$ & $\begin{array}{c}\text { Significant improvements were observed } \\
\text { between groups in spasticity }(p=0.016) \text {. } \\
\text { Not significant results were found in other } \\
\text { outcomes }\end{array}$ \\
\hline $\begin{array}{l}\text { Deutz et al., } 2017 \\
\text { [24] }\end{array}$ & $\begin{array}{l}\text { IG: }(n=35) \\
\text { CG: }(n=38)\end{array}$ & $\begin{array}{l}\text { IG: HPT } \\
\text { CG: PT }\end{array}$ & 1-2 times/week & ND & 16-20 weeks & $\begin{array}{l}\text { Gross motor function and } \\
\text { quality of life }\end{array}$ & $\begin{array}{l}\text { GMFM-66 } \\
\text { KIDSCREEN-27 } \\
\text { questionnaire } \\
\text { CHQ }\end{array}$ & $\begin{array}{l}\text { Improvements were observed in } \\
\text { GMFM- } 66 \text { dimension E for IG }(p=0.02) \\
\text { compared to CG. Not significant results } \\
\text { were found in quality of life }\end{array}$ \\
\hline $\begin{array}{l}\text { Lucena-Antón et al., } \\
\quad 2018 \text { [5] }\end{array}$ & $\begin{array}{l}\text { IG: }(n=22) \\
\text { CG: }(n=22)\end{array}$ & $\begin{array}{l}\text { IG: HPT } \\
\text { CG: PT }\end{array}$ & $\begin{array}{l}\text { IG: Once a week } \\
\text { CG: } 2 \\
\text { times/week }\end{array}$ & $45 \mathrm{~min}$ & 12 weeks & Hip adductors spasticity & MAS & $\begin{array}{l}\text { Significant results were obtained between } \\
\text { groups for IG in spasticity ( } p=0.04 \text { for left } \\
\text { adductors and } p=0.047 \text { for right } \\
\text { adductors) }\end{array}$ \\
\hline
\end{tabular}

CG: control group; CHQ: Child Health Questionnaire; EMG: Electromyography; GMFM: Gross Motor Function Measure; HPT: Hippotherapy; IG: Intervention group; MAS: Modified Ashworth Scale; MFRT Modified Functional Reach Test; Min: Minutes; ND: not described; PBS Pediatric Balance Scale; PEDI-FSS: Pediatric Evaluation of Disability Inventory-Functional Skills Scale; PT: Physical therapy. SAS Sitting Assessment Scale; PDM Multifunction Force Measure Plate; SD: Standard deviation; SEMG: Surface Electromyography. 


\subsection{Meta-Analysis of the Study Groups}

The groups were created according to the measuring instrument used to assess the gross motor function. Accordingly, seven groups were set up: (i) GMFM-66 total scores; (ii) GMFM-88 total scores; and (iii-vii) GMFM-88 dimensions A-E.

The gross motor function measure (GMFM) is commonly used in neurological rehabilitation to assess the gross motor function in subjects with CP. The GMFM-66 scale is an updated version of GMFM-88. It includes 66 of the original 88 items providing more information to encourage the goal setting process [11]. Both scales include different items that assess how much of an activity can be carried out rather than the quality of performing the activities [25]. Both versions have been validated to evaluate changes in children with CP. A higher score is an indicator of better gross motor function [26].

Two studies analyzed the differences in gross motor function using the GMFM-66. The overall result of this study group was favorable (Figure 2).

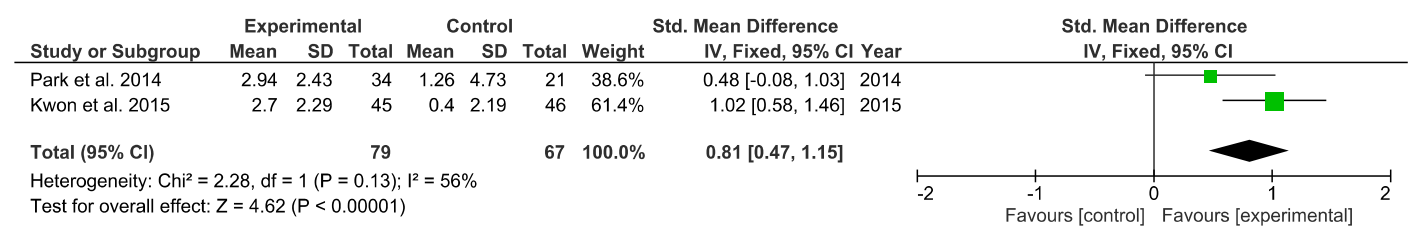

Figure 2. Forest plot for gross motor function measured by the GMFM-66 scale.

Regarding the GMFM-88 scale, it is divided into five dimensions (A: lying and rolling, B: sitting, $\mathrm{C}$ : crawling and kneeling, D: standing, and E: walking, running, and jumping). The total score ranges from 0 to 100. For the GMFM-88 total score, the overall result of the meta-analysis was not conclusive (Figure 3).

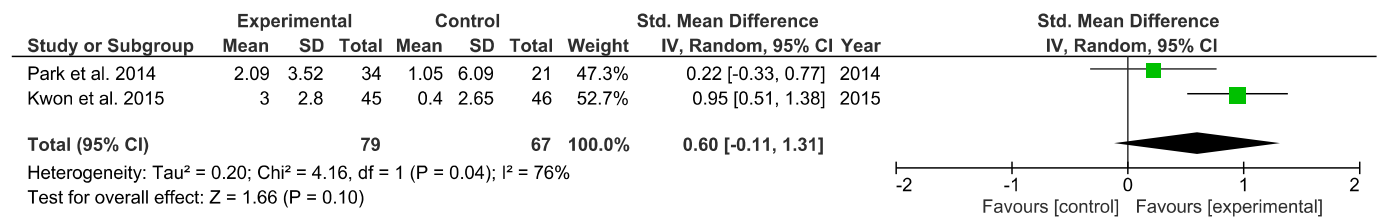

Figure 3. Forest plot for gross motor function measured by the GMFM-88 total scores.

Regarding the different dimensions included in the GMFM-88 scale, the overall result of the meta-analysis was favorable in GMFM- 88 dimensions A, B, and E, while the overall result of the meta-analysis was inconclusive for GMFM-88 dimensions C and D. The results are shown in Figures 4-8.

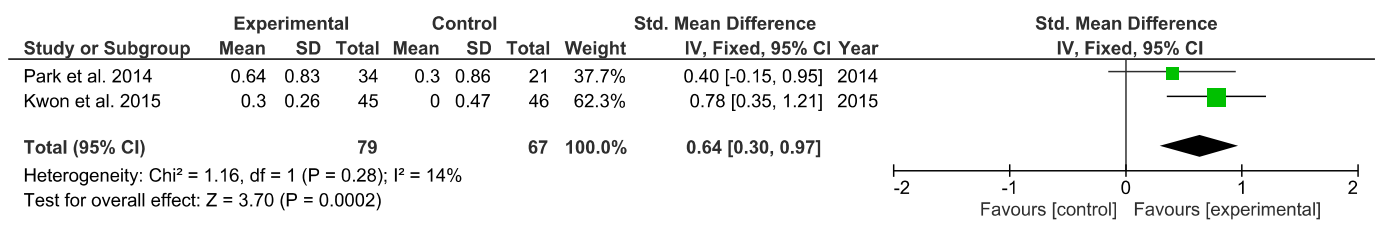

Figure 4. Forest plot for GMFM-88 Dimension A.

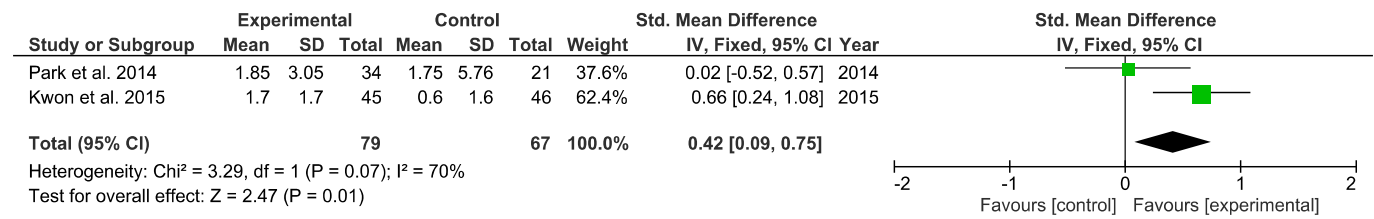

Figure 5. Forest plot for GMFM-88 Dimension B. 


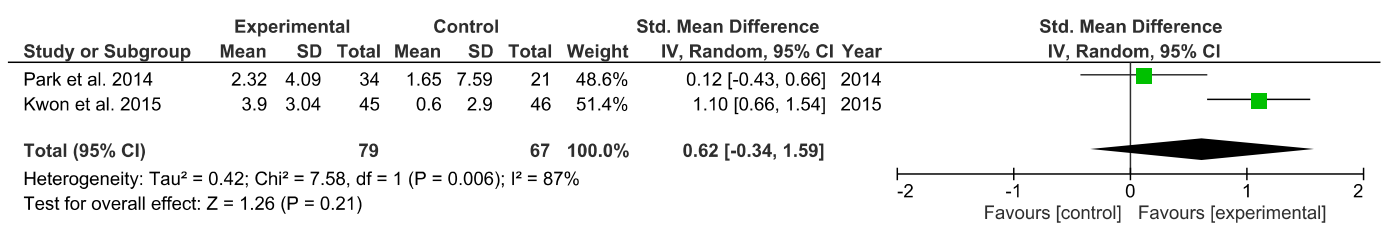

Figure 6. Forest plot for GMFM-88 Dimension C.

\begin{tabular}{|c|c|c|c|c|c|c|c|c|c|c|c|}
\hline \multirow[b]{2}{*}{ Study or Subgroup } & \multicolumn{3}{|c|}{ Experimental } & \multicolumn{3}{|c|}{ Control } & \multicolumn{2}{|r|}{ Std. Mean Difference } & \multirow{2}{*}{\multicolumn{3}{|c|}{$\begin{array}{l}\text { Std. Mean Difference } \\
\text { IV, Random, } 95 \% \mathrm{Cl}\end{array}$}} \\
\hline & Mean & SD & Total & Mean & SD & Total & Weight & IV, Random, $95 \%$ CI Year & & & \\
\hline Park et al. 2014 & 3 & 5.75 & 34 & 0.6 & 9.74 & 21 & $48.6 \%$ & $0.32[-0.23,0.86] 2014$ & & 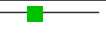 & \\
\hline Kwon et al. 2015 & 5.6 & 4.98 & 45 & -0.6 & 4.82 & 46 & $51.4 \%$ & $1.25[0.80,1.71] 2015$ & & & \\
\hline Total $(95 \% \mathrm{Cl})$ & & & 79 & & & 67 & $100.0 \%$ & $0.80[-0.12,1.72]$ & & & \\
\hline $\begin{array}{l}\text { Heterogeneity: } \mathrm{Tau}^{2}= \\
\text { Test for overall effect: }\end{array}$ & $\begin{array}{l}0.38 ; C h \\
z=1.70\end{array}$ & $\begin{array}{l}i^{2}=6.7 \\
(P=0 . C\end{array}$ & & $=1(P=$ & 0.009); & $I^{2}=85$ & & & $\begin{array}{c}1 \\
-1 \\
\text { Favours [control] }\end{array}$ & Favours [ex & $\begin{array}{l}1 \\
\text { experimental] }\end{array}$ \\
\hline
\end{tabular}

Figure 7. Forest plot for GMFM-88 Dimension D.

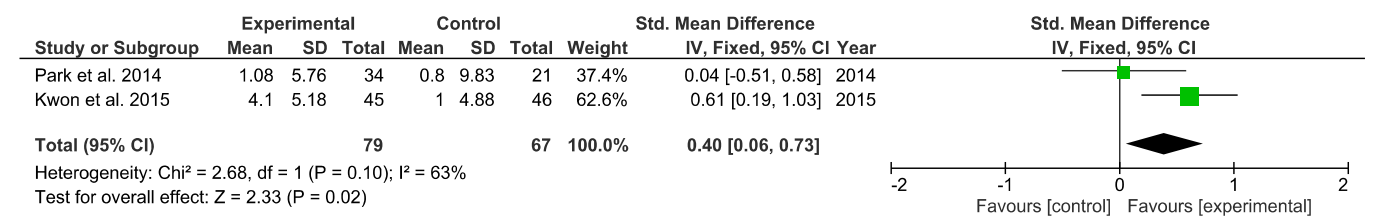

Figure 8. Forest plot for GMFM-88 Dimension E.

\section{Discussion}

The objective of this systematic review and meta-analysis of RCTs was to analyze the effectiveness of HPT interventions on improving gross motor function in subjects with CP. A total of ten RCTs were analyzed in the systematic review, involving 452 participants. In view of our results, we could conclude that HPT could be an effective intervention to improve gross motor function in children with CP.

From a clinical perspective, the findings obtained in the present review suggest that HPT stands for an emerging intervention in neurological rehabilitation, which could be used in addition to neurodevelopmental based methods. The findings on the GMFM-66 scale and GMFM-88 dimensions A, B, and E showed that HPT interventions had significant improvements on gross motor function and, more specifically, on the ability to perform lying and rolling, sitting, and walking. We can hypothesize that the rhythmic and symmetrical movement of the horse could stimulate the proprioception and balance reactions. Furthermore, according to Casady and Nichols-Larsen [27], HPT could stimulate the motor learning and subjects could be able to transfer the movement patterns learned from HPT to other usual environments. According to Bertoti [28], and considering that three of four studies [17,18,20,23] did obtain significant effects on balance and two studies $[5,23]$ reported significant effects on the spasticity of hip adductors, we can suggest that the positive effects obtained on balance and muscle spasticity contributed to improvements in the functional outcomes and, thus, to the significant results obtained in the GMFM-66 and GMFM-88 dimensions.

Regarding the intervention characteristics, most studies included more than 30 participants, a high number considering the difficulty to recruit patients with CP. All intervention groups received HPT in addition to physical therapy, and all of them carried out their HPT interventions through a walking pace, except for Alemdaroğlu et al. [23] and Deutz et al. [24] that did not specify it. Most studies used protocols with 8-12 weeks as the total duration and two times/week as the frequency. The session duration used in the studies was around $30 \mathrm{~min}$, with unusual interventions of more than $45 \mathrm{~min}$. The effects found were similar and several authors suggested that longer durations could cause fatigue in children, which was not positive for achieving the intended improvements. Therefore, we can suggest that HPT interventions based on 8-12 week programs with sessions of 30-45 min two times a week could be proper for children with $\mathrm{CP}$ to recover motor function.

Concerning the methodological quality of the studies included in the present review, the main limitation was found in the application of double-blind. Blinding of the participants and therapists 
was not possible in most studies due to the unconcealable nature of the intervention. In addition, the concealed allocation was only possible in two studies $[16,19]$ and the assessor blinding was carried out by four studies $[5,16,17,24]$. Nevertheless, the overall quality of the studies was acceptable.

Our results matched with the findings of the systematic review conducted by Whalen and Case-Smith [12] in 2012, in which they stated that HPT could produce benefits on gross motor function in children with $\mathrm{CP}$, but the authors highlighted that the evidence was limited. Other findings were found in different pathologies, such as Down syndrome and autism disorder. De Miguel Rubio et al. [29] suggested that HPT could not be effective to improve gross motor function in subjects with Down syndrome, and Srinivasan et al. [30] analyzed the effects of HPT interventions in subjects with autism disorder, obtaining positive effects on motor skills.

The present systematic review presented some limitations. Potential useful articles that were indexed in other scientific databases could not be included. In addition, the lack of long-term follow-up and the heterogeneity of the protocols suggests the need to unify the HPT intervention programs, specifically, in subjects with CP. Moreover, despite assessing the same outcomes between the different studies included in the review, the statistical comparison was not always possible due to studies used different scales and measuring instruments to assess the clinical differences. Thus, only two studies were included in the meta-analysis. Therefore, the results obtained should be taken with caution since a limited number of studies was analyzed.

\section{Conclusions}

In conclusion, we could state that HPT interventions were effective to improve gross motor function in subjects with CP. Specifically, favorable results were obtained in the GMFM-66 total scores and GMFM-88 dimensions A, B, and E. Furthermore, positive effects have been showed on balance recovery and muscle spasticity reduction.

Despite the different HPT protocols used, evidence shows that 30-45 min sessions, twice weekly for 8-12 weeks, could produce significant effects on gross motor function in children with CP.

This study can be helpful in neurological rehabilitation of children with CP using HPT interventions, as well as by providing key factors to determine which specific factors of the HPT protocols have a greater weight to achieve the desired effects in future interventions. Nevertheless, it will be necessary to carry out more randomized controlled trials with larger sample sizes and specified protocols.

Author Contributions: Conceptualization and methodology: L.D.G.-S., I.C.-B., J.A.M.-M. and D.L.-A.; statistical analysis: A.S.; writing-original draft preparation: L.D.G.-S., D.L.A., A.S. and J.A.M.-M.; writing-review and editing: D.L.-A., J.A.M.-M. and I.C.-B. All authors have read and agreed to the published version of the manuscript.

Funding: This research was funded by the Department of Nursing and Physiotherapy (University of Cadiz).

Acknowledgments: The authors are grateful for the funding from the Department of Nursing and Physiotherapy (University of Cadiz).

Conflicts of Interest: The authors declare no conflict of interest.

\section{References}

1. Petersen, R.; Procter, C.; Donald, K.A. Assessment and Management of the Child with Cerebral Palsy. In Clinical Child Neurology; Springer International Publishing: Cham, Switzerland, 2020; pp. 175-203.

2. Longo, E.; Regalado, I.C.R.; Galvão, E.R.V.P.; Ferreira, H.N.C.; Badia, M.; Baz, B.O. I Want to Play: Children With Cerebral Palsy Talk About Their Experiences on Barriers and Facilitators to Participation in Leisure Activities. Pediatr. Phys. Ther. 2020, 32, 190-200. [PubMed]

3. Novak, I.; Morgan, C.; Adde, L.; Blackman, J.; Boyd, R.N.; Brunstrom-Hernandez, J.; Cioni, G.; Damiano, D.; Darrah, J.; Eliasson, A.-C.; et al. Early, Accurate Diagnosis and Early Intervention in Cerebral Palsy. JAMA Pediatr. 2017, 171, 897. [CrossRef] [PubMed]

4. Dominguez-Romero, J.G.; Molina-Aroca, A.; Moral-Munoz, J.A.; Luque-Moreno, C.; Lucena-Anton, D. Effectiveness of mechanical horse-riding simulators on postural balance in neurological rehabilitation: Systematic review and meta-analysis. Int. J. Environ. Res. Public Health 2020, 17, 165. [CrossRef] [PubMed] 
5. Lucena-Anton, D.; Rosety-Rodríguez, I.; Moral-Munoz, J.A. Effects of a hippotherapy intervention on muscle spasticity in children with cerebral palsy: A randomized controlled trial. Complement Ther. Clin Pract. 2018, 31, 188-192. [CrossRef]

6. Koca, T.T. What is hippotherapy? The indications and effectiveness of hippotherapy. North Clin. Istanbul. 2016, 2, 247-252. [CrossRef]

7. Novak, I.; Morgan, C.; Fahey, M.; Finch-Edmondson, M.; Galea, C.; Hines, A.; Langdon, K.; Mc Namara, M.; Paton, M.C.B.; Popat, H.; et al. State of the Evidence Traffic Lights 2019: Systematic Review of Interventions for Preventing and Treating Children with Cerebral Palsy. Curr. Neurol. Neurosci. Rep. 2020, 20, 3. [CrossRef]

8. Alonso, P.M. Physiotherapy interventions through hippotherapy in the treatment of cerebral palsy. A literature review. Rehabilitación 2020, 54, 96-106.

9. Martín-Valero, R.; Vega-Ballón, J.; Perez-Cabezas, V. Benefits of hippotherapy in children with cerebral palsy: A narrative review. Eur. J. Paediatr. Neurol. 2018, 22, 1150-1160. [CrossRef]

10. Zadnikar, M.; Kastrin, A. Effects of hippotherapy and therapeutic horseback riding on postural control or balance in childrenwith cerebral palsy: A meta-analysis. Dev. Med. Child Neurol. 2011, 53, 684-691. [CrossRef]

11. Alotaibi, M.; Long, T.; Kennedy, E.; Bavishi, S. The efficacy of GMFM-88 and GMFM-66 to detect changes in gross motor function in children with cerebral palsy (CP): A literature review. Disabil. Rehabil. 2014, 36, 617-627. [CrossRef]

12. Whalen, N.C.; Case-Smith, J. Therapeutic effects of horseback riding therapy on gross motor function in children with cerebral palsy: A Systematic Review. Phys. Occup. Ther. Pediatr. 2012, 32, 229-242. [CrossRef] [PubMed]

13. Hutton, B.; Catalá-López, F.; Moher, D. The PRISMA statement extension for systematic reviews incorporating network meta-analysis: PRISMA-NMA. Med. Clin. 2016, 147, 262-266. [CrossRef]

14. Maher, C.G.; Sherrington, C.; Herbert, R.D.; Moseley, A.M.; Elkins, M. Reliability of the PEDro Scale for Rating Quality of Randomized Controlled Trials. Phys. Ther. 2003, 83, 713-721. [CrossRef] [PubMed]

15. Moseley, A.M.; Herbert, R.D.; Sherrington, C.; Maher, C.G. Evidence for physiotherapy practice: A survey of the Physiotherapy Evidence Database (PEDro). Aust. J. Physiother. 2002, 48, 43-49. [CrossRef]

16. McGibbon, N.H.; Benda, W.; Duncan, B.R.; Silkwood-Sherer, D. Immediate and long-term effects of Hippotherapy on symmetry of adductor muscle activity and functional ability in children with spastic cerebral palsy. Arch Phys. Med. Rehabil. 2009, 90, 966-974. [CrossRef]

17. Kwon, J.-Y.; Chang, H.J.; Yi, S.-H.; Lee, J.Y.; Shin, H.-Y.; Kim, Y.-H. Effect of hippotherapy on gross motor function in children with cerebral palsy: A randomized controlled trial. J. Altern Complement Med. 2015, 21, 15-21. [CrossRef]

18. Matusiak-Wieczorek, E.; Małachowska-Sobieska, M.; Synder, M. Influence of Hippotherapy on Body Balance in the Sitting Position Among Children with Cerebral Palsy. Ortop. Traumatol. Rehabil. 2016, 18, 165-175. [CrossRef]

19. Benda, W.; McGibbon, N.H.; Grant, K.L. Improvements in muscle symmetry in children with cerebral palsy after equine-assisted therapy (Hippotherapy). J. Altern Complement Med. 2003, 9, 817-825. [CrossRef]

20. Kang, H.; Jung, J.; Yu, J. Effects of Hippotherapy on the Sitting Balance of Children with Cerebral Palsy: A Randomized Control Trial. J. Phys. Ther. Sci. 2012, 24, 833-836. [CrossRef]

21. El-Meniawy, G.H.; Thabet, N.S. Modulation of back geometry in children with spastic diplegic cerebral palsy via hippotherapy training. Egypt J. Med. Hum. Genet. 2012, 13, 63-71. [CrossRef]

22. Park, E.S.; Rha, D.-W.; Shin, J.S.; Kim, S.; Jung, S. Effects of Hippotherapy on gross motor function and functional performance of children with cerebral palsy. Yonsei Med. J. 2014, 55, 1736-1742. [CrossRef] [PubMed]

23. Alemdaroğlu, E.; Oken, O.; Ucan, H.; Ersöz, M.; Köseoğlu, B.F.; Kapıcıoğlu, M. İsmail S.; Yanıkoğlu, I. Horseback riding therapy in addition to conventional rehabilitation program decreases spasticity in children with cerebral palsy: A small sample study. Complement Ther. Clin. Pract. 2016, 23, 26-29. [CrossRef]

24. Deutz, U.; Heussen, N.; Weigt-Usinger, K.; Leiz, S.; Raabe, C.; Polster, T.; Steinbüchel, D.; Moll, C.; Lücke, T.; Krägeloh-Mann, I.; et al. Impact of Hippotherapy on Gross Motor Function and Quality of Life in Children with Bilateral Cerebral Palsy: A Randomized Open-Label Crossover Study. Neuropediatrics 2018, 49, $185-192$. [CrossRef] [PubMed] 
25. Russell, D.; Rosenbaum, P.; Avery, L.; Lane, M. Gross Motor Function Measure (GMFM-66 and GMFM-88) User's Manual: Clinics in Development Medicine; Mac Keith Press: London, UK, 2002.

26. Wei, S.; Wang, S.J.; Liao, Y.G.; Hong, Y.; Xu, X.J.; Shao, X.M. Reliability and validity of the GMFM-66 in 0- to 3-year-old children with cerebral palsy. Am. J. Phys. Med. Rehabil. 2006, 85, 141-147. [CrossRef] [PubMed]

27. Casady, R.L.; Nichols-Larsen, D.S. The effect of hippotherapy on ten children with cerebral palsy. Pediatr. Phys. Ther. 2004, 16, 165-172. [CrossRef]

28. Bertoti, D.B. Effect of therapeutic horseback riding on posture in children with cerebral palsy. Phys. Ther. 1988, 68, 1505-1512. [PubMed]

29. De Miguel, A.; De Miguel, M.D.; Lucena-Anton, D.; Rubio, M.D. Effects of hypotherapy on the motor function of persons with Down's syndrome: A systematic review. Rev. Neurol. 2018, 67, 233-241.

30. Srinivasan, S.M.; Cavagnino, D.T.; Bhat, A.N. Effects of Equine Therapy on Individuals with Autism Spectrum Disorder: A Systematic Review. Rev. J. Autism Dev. Disord. 2018, 5, 156-175. [CrossRef]

(C) 2020 by the authors. Licensee MDPI, Basel, Switzerland. This article is an open access article distributed under the terms and conditions of the Creative Commons Attribution (CC BY) license (http://creativecommons.org/licenses/by/4.0/). 